\title{
Application of Design of Experiment and Design Space (DOE-DS) Methodology for the HPLC Separation of Panax Notoginseng Saponins
}

\author{
Shengyun Dai, Bing Xu*, Gan Luo, Jianyu Li, Zhong Xue, Xinyuan Shi and Yanjiang Qiao*
}

Beijing University of Chinese Medicine, Key Laboratory of TCM-information Engineering of State Administration of TCM, South of Wangjing Middle Ring Road, Chaoyang District, Beijing City, China, 100102

\begin{abstract}
Abatract: Panax Notoginseng Saponins (PNS), extracted from the roots of the common TCM Panax notoginseng (Burk.) F.H.Chen, consist of notoginsenoside $\mathrm{R}_{1}$, ginsenoside $\mathrm{Rg}_{1}, \mathrm{Re}, \mathrm{Rb}_{1}, \mathrm{Rd}$ and many other chemicals which haven't been identified. Due to its popular pharmacological effects, it is of importance to control the quality. This study combines design of experiments and design space methodology to optimize the HPLC separation of PNS. Three common chromatographic parameters (i.e. the temperature, the initial proportion of acetonitrile and the gradient slope) were selected to construct a Box-Behnken design which consisted of 17 experiments. A quadratic model that was built from the experimental result was used to construct the design space. The optimal separation was predicted at temperature $20^{\circ} \mathrm{C}$, with a gradient starting at $15 \%$ of acetonitrile and a gradient slope of $0.55 \% / \mathrm{min}$. Accuracy profile approach was employed to validate the established HPLC method. The results clearly showed that quality by design methodology could be effectively applied to optimize the HPLC chromatographic conditions for the analysis of PNS.
\end{abstract}

Keywords: Panax notoginseng saponins, quality by design, design of experiments, design space, accuracy profile.

\section{INTRODUCTION}

Nowadays, though chemical drugs have a strong impact on the world's drug market, Traditional Chinese Medicine (TCM) still holds an important place to cure diseases in many Asian countries. The root of Panax notoginseng, called Sanqi or Tianqi in China, is a popular medical material which is well known for its efficacy in promoting blood circulation, removing blood stasis. And it is important to control the quality of PNS to achieve its clinical safety and efficacy for PNS is the main component in Panax notoginseng. Most of the studies about the quality control of PNS are based on HPLC, and the optimal chromatographic conditions were obtained using one factor at a time (OFAT) experiments. However, the OFAT method cannot reveal the interaction among different factors. Recently, Quality-by-design $(\mathrm{QbD})$ concept advocated by ICH and FDA has been introduced into the fields of chromatographic analysis[1,2]. QbD contained several parts, such as design of experiments (DoE) and design space (DS). Design of experiments (DoE) methodology was used to investigate the factors effects and the interactions between them by modifying multiple factors at a time [3]. Design space is defined as the "multidimensional combination and interaction of input variables that have been demonstrated to provide assurance of quality" [4]. Therefore, combined DoE with DS, a robust analytical method can be developed to satisfy the movement of HPLC parameters.

\footnotetext{
*Address correspondence to these authors at the Beijing University of Chinese Medicine, Key Laboratory of TCM-information Engineering of State Administration of TCM, South of Wanging Middle Ring Road, Chaoyang District, Beijing, China, 100102; E-mail: btcm@163.com; yjqiao@263.net
}

The main objective of this work is to develop a method for the separation of the 5 components in PNS by design of experiments and design space based on HPLC, and then the method was validated by accuracy profile methodology based on total errors theory [5].

\section{MATERIALS}

\subsection{Reagents}

Notoginsenoside $R_{1}$ reference standard was purchased from Nature Standard Biological Technology Co., Ltd. (lot:STA-25613013), ginsenoside $\mathrm{Rg}_{1}, \mathrm{Re}, \mathrm{Rb}_{1}$ were purchased from Sichuan Weikeqi Biological Technology Co., Ltd. (lot: 110626, 110819, 110723, respectively), and ginsenoside Rd was purchased from Jiangxi bencaotiangong Science and Technology Co., Ltd.(lot:ROH-130912). The reagents were purchased as follows: both methanol HPLC grade and acetonitrile HPLC grade from Fisher scientific (Erembodegem,Belgium), distilled water.

\subsection{Extraction}

The sample Panax Notoginseng Saponins extraction was purchased from Jiangxi bencaotiangong Science and Technology Co., Ltd(lot:20130825).

\subsection{Apparatus}

Chromatographic separations were performed with a reversed phase $\mathrm{C} 18$ column $(150 \mathrm{~mm} \times 4.6 \mathrm{~mm}$ i.d.; particle size: $5 \mu \mathrm{m}$ ), a HPLC agilent 1100 series separation module, coupled to a DAD spectrophotometric detector. 


\section{EXPERIMENTAL}

\subsection{Sample preparation}

The sample was dissolved to $10 \mathrm{~mL} 70 \%$ methanol, filtered through a 0.45 um filter. Finally, $10 \mathrm{ul}$ of this solution were injected into the HPLC system.

\subsection{Experimental design}

DOE is considered a time-efficient and cost-efficient technique used to simultaneously identify the effects of multiple factors on results [6]. Box-Behnken Design was chosen to complete method optimization which was one of the design pattern of RSM (Response Surface Method). The method optimization involved 3 chromatographic factors: temperature, gradient slope and the initial concentration of acetonitrile and all of them was studied with 3 levels(see Table 1). 17 experiments of the three parameters have been tried according to the requirements of Box-Behnken Design.

\subsection{Chromatographic conditions}

The mobile phase consisted of acetonitrile and water. Experiments were carried out in gradient mode. The temperature of the column was adjust to $20^{\circ} \mathrm{C}, 24^{\circ} \mathrm{C}, 28^{\circ} \mathrm{C}$, flow rate was $1 \mathrm{ml} / \mathrm{min}$ and the detection was performed at a wavelength of $203 \mathrm{~nm}$. Peaks were fitted by Origin 8 software as some peaks may not be separated under some chromatographic conditions.

\subsection{Method validation}

Accuracy, precision, limit of detection, limit of quantitation, linearity and range is the common parameters to estimate the method validation. The classical approaches are to investigate these parameters separately which need much time and resources. AOAC International defines a validated method as a method that is fit for its intended purpose and accuracy profile [7] is one of the methods. Accuracy profile has become an effective decision tool to assess the validity of analytical methods which based on the three most important validation parameters: trueness, precision and range. The validation of the method was done during three days to evaluate time-dependent intermediate precision. Four concentration levels were selected to establish the curve. Each level of the validation standards was analyzed independently in triplicates.

\section{RESULTS AND DISCUSSION}

\subsection{Experiment design}

The first step is to verify the validity of the model for each response. In total,5 peaks were identified as Notoginsenoside $\mathrm{R}_{1}$, ginsenoside $\mathrm{Rg}_{1}, \mathrm{Re}, \mathrm{Rb}_{1}, \mathrm{Rd}$ (from left to right in the chromatomap). As depicted in Fig. (1), the adequacy between the sample retention times and the standard is good. Factors which have the highest effects on the response will be selected. Usually, the chromatographic resolution(Rs) between two adjacent peaks is modeled to assess separation, and the least Rs of two peaks was regarded as the critical peaks when there are many Rs in one chromatomap. The objective of optimization of the HPLC method is to let the critical Rs meet the Pharmacopoeia requirements. Besides, another objective is to shorten the running time $\left(t_{R}\right)$. After comparing different models, the quadratic model was selected because it has the largest "Adjusted R-Squared" and the "Predicted R-Squared". The critical peaks are the ginsenoside

Table 1. Chromatographic parameters studied for the method optimization. (B\%: the initial concentration of acetonitrile)

\begin{tabular}{|c|c|c|c|}
\hline Parameter & & Levels \\
\hline \hline Temperature $\left[{ }^{\circ} \mathrm{C}\right]$ & 20 & 24 & 28 \\
\hline Gradient slope & 0.4 & 0.6 & 10 \\
\hline $\mathrm{B}[\%]$ & 5 & 0.8 & 15 \\
\hline
\end{tabular}
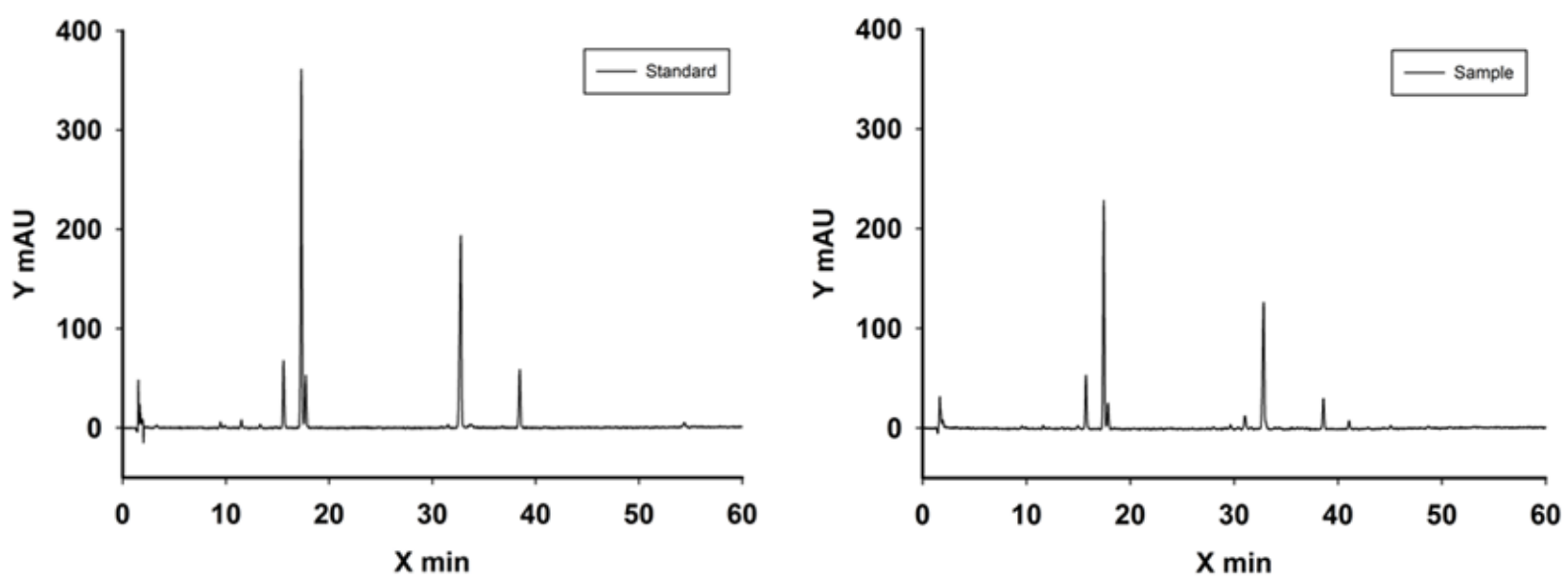

Fig. (1). The adequacy between the sample retention times and the standard ones is good. 
$\mathrm{Rg}_{1}$ and ginsenoside $\mathrm{Re}$ in the separation of PNS. These responses were modeled by quadratic equations (see Eq. 1)

$$
\begin{aligned}
& R_{S}=5.16-0.0629 \quad T-6.36 \quad G+(6.40 E-003) \quad B+ \\
& 0.0438 \quad T \quad G+(2.71 E-007) \quad T \quad B+0.025 \quad G \quad B- \\
& (3.12 E-005) T^{2}+2.49 G^{2}-(1.22 E-003) \quad B^{2}
\end{aligned}
$$

where Rs represents the separation of the critical peaks. $\mathrm{T}$ (temperature), $\mathrm{G}$ (gradient), $\mathrm{B}$ are the factors of the design of experiments. Eq. 1 regard Rs as the response to build mathematic model, and $t_{R}$ can also be seen as one response to build a quadratic model as Rs. The "Adjusted R-Squared" of the model is 0.987 and the "Predicted R-Squared" is 0.9975 indicating this model is of significance to navigate the design space. The DS corresponds to a region of the experimental domain where the probability to attain Rs $>1.5$ and $t_{R}>b(b$ represents a certain time can be set by end user)

The overlay plot shows the Design Space of the method (see Fig. 2). The colored part represents the interaction of the two responses. The operating conditions changed included in the bright yellow zone will not cause the changing of the method. That is to say, the method is not needed to be validated under the condition. However, there will be some risk if the operating conditions changed included in the dark yellow zone. The dark yellow zone was built based on 95\% confidence intervals. Besides, the optimal separation was predicted at $20^{\circ} \mathrm{C}$, with a gradient starting at $15 \%$ of acetonitrile and a gradient slope of $0.55 \% / \mathrm{min}$ from the Design Space(the blue dot in the Fig. 2). This optimal condition will be used at method validation.

\subsection{Method validation}

Trueness is related to the systematic error from its definition. It is expressed in relative bias(\%) at each concentration level of the 3 repeats for the 3 days. Relative bias was less than $5 \%$ for the 5 components (see Table 2 , take notoginsenoside $\mathrm{Rg}_{1}$ as an example, the follows are the same) of the four levels showing the adequate trueness of the method except the least concentration level. Relative bias for the least concentration is more than $5 \%$ means the least concentration cannot satisfy the requirement of trueness.

Precision is related to the accidental error from its definition. It is expressed in relative standard deviation (RSD\%) values for repeatability and intermediate precision $[8,9]$. $\operatorname{RSD}(\%)$ for repeatability and intermediate precision did not exceed 2\% (see Table 2) for the 5 components of the four levels indicating the accidental error can be accepted of the method except the least concentration level. RSD (\%) for the least concentration is more than 3\% means the least concentration level cannot satisfying the requirements of precision as well.

Accuracy is related to the total errors containing systematic and accidental error of the test results. Fig. (3) (1) shows that the $90 \% \beta$-expectation upper and lower tolerance limits are totally included in the acceptance limits set at $\pm 10 \%$ for the whole concentration range except the first concentration of ginsenoside $R_{1}$. For notoginsenoside $R_{1}$, ginsenoside $\mathrm{Re}$, ginsenoside $R b_{1}$ and ginsenoside $\mathrm{Rd}$, as illustrated in Fig. (3) (2)(3)(4)(5), their accuracy profiles show that the relative upper and lower $90 \% \beta$-expectation tolerance limits are totally included inside the acceptance limits set at $\pm 5 \%$ except the least concentration level. Consequently, the method can be considered as accurate between 0.1484 and $0.486 \mathrm{mg} / \mathrm{mL}$ for ginsenoside $R_{1}$, between 0.558 and $2.178 \mathrm{mg} / \mathrm{mL}$ for ginsenoside $\mathrm{Rg}_{1}$, between 0.104 and $0.273 \mathrm{mg} / \mathrm{mL}$ for ginsenoside Re, between 0.375 and $2.156 \mathrm{mg} / \mathrm{mL}$ for ginsenoside $\mathrm{Rb}_{1}$ and between 0.123 and $0.306 \mathrm{mg} / \mathrm{mL}$ for ginsenoside Rd (see Table 2).

Limits of quantitation (LOQ) were $0.148 \mathrm{mg} / \mathrm{mL}, 0.558$ $\mathrm{mg} / \mathrm{mL}, 0.104 \mathrm{mg} / \mathrm{mL}, 0.375 \mathrm{mg} / \mathrm{mL}, 0.123 \mathrm{mg} / \mathrm{mL}$ (see Table 2) of the 5 components respectively. For the LOQ it was

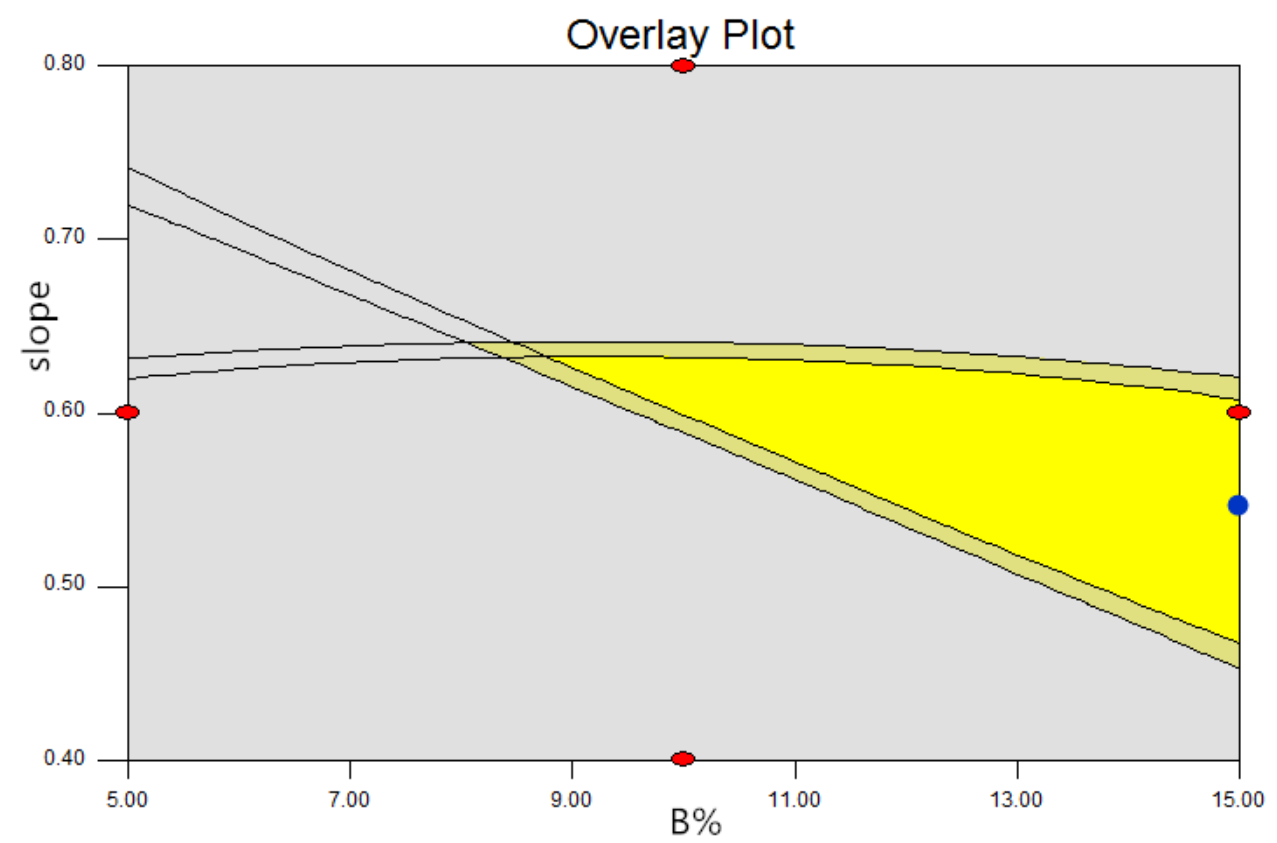

Fig. (2). The Design Space of the method. The blue dot is the optimized conditions. 
Table 2. Validation results of the HPLC-UV method for the determination of the notoginsenoside Rg1 (3 series, 3 repetitions).

\begin{tabular}{|c|c|c|c|c|}
\hline & $\begin{array}{l}\text { Validation } \\
\text { parameter }\end{array}$ & & & \\
\hline \multirow{12}{*}{ notoginsenoside $R_{1}$} & \multirow{4}{*}{ Trueness } & Concentration $(\mathrm{mg} / \mathrm{mL})$ & Relative bias (\%) & \\
\hline & & 0.0405 & -4.374 & \\
\hline & & 0.203 & -3.925 & \\
\hline & & 0.486 & -3.707 & \\
\hline & \multirow[t]{2}{*}{ Precision } & Concentration $(\mathrm{mg} / \mathrm{mL})$ & Repeatability (RSD\%) & $\begin{array}{l}\text { Intermediate precision } \\
(\mathrm{RSD} \%)\end{array}$ \\
\hline & & 0.0405 & 3.810 & 3.937 \\
\hline & \multirow[t]{5}{*}{ Accuracy } & Concentration $(\mathrm{mg} / \mathrm{mL})$ & $\begin{array}{l}90 \% \beta \text {-expectation lower and upper } \\
\text { tolerance limits of the relative error }(\%)\end{array}$ & \\
\hline & & 0.0405 & {$[-12.23,3.478]$} & \\
\hline & & 0.203 & {$[-6.949,-0.900]$} & \\
\hline & & 0.324 & {$[-7.306,-2.374]$} & \\
\hline & & 0.486 & {$[-5.669,-1.744]$} & \\
\hline & Range & \multicolumn{3}{|c|}{$0.1484-0.4865 \mathrm{mg} / \mathrm{mL}$} \\
\hline
\end{tabular}

obtained from the accuracy profile (see Fig. 3) where the crossing point of accuracy limits and $90 \% \beta$-expectation limits. The range is the interval between LOQ and the last concentration where the procedure achieves adequate accuracy.

In order to demonstrate the linearity of method, a linear regression model is fitted on the results as a function of the introduced concentrations in order to obtain linearity equation. The linearity equation of the 5components are $\mathrm{Y}=-0.0007604+0.9617 \mathrm{X}, \quad \mathrm{Y}=0.001404+0.9818 \mathrm{X}, \quad \mathrm{Y}=0.00$ $1805+0.9863 X, Y=0.004275+0.9905 X, Y=-0.001496+1.00$ $5 \mathrm{X}$. All the $\mathrm{r}$ value of the 5 linearity equation are bigger than 0.9992 (see Table 2) which demonstrate the method has a good relationship between introduced and calculated concentration using $\beta$-expectation tolerance interval approach.

\section{CONCLUSION}

A novel analytical method for the separation of notoginsenoside $\mathrm{R}_{1}$, ginsenoside $\mathrm{Rg}_{1}, \mathrm{Re}, \mathrm{Rb}_{1}, \mathrm{Rd}$ in PNS was developed using DoE-DS methodology based on HPLC.
Interactions of the three critical parameters are showed on the design space. The method developed and validated was then successfully applied to quantify these five compounds.

Results demonstrated that the QbD approach can provide a better understanding of the critical parameters of a method and allows greater flexibility especially when time and resource are under constraints.

\section{CONFLICT OF INTEREST}

The authors confirm that this article content has no conflict of interest.

\section{ACKNOWLEDGEMENTS}

The author is grateful for the financial supports from the Joint Development Program Supported by Beijing Municipal Education Commission - Key Laboratory Construction Project (Study on the Integrated Modeling and Optimization Technology of the Chained Pharmaceutical Process of Chinese Medicine Products) and the Innovation Team of TCM information Engineering of Beijing University of Chinese Medicine(No. 500102/0100605158). 

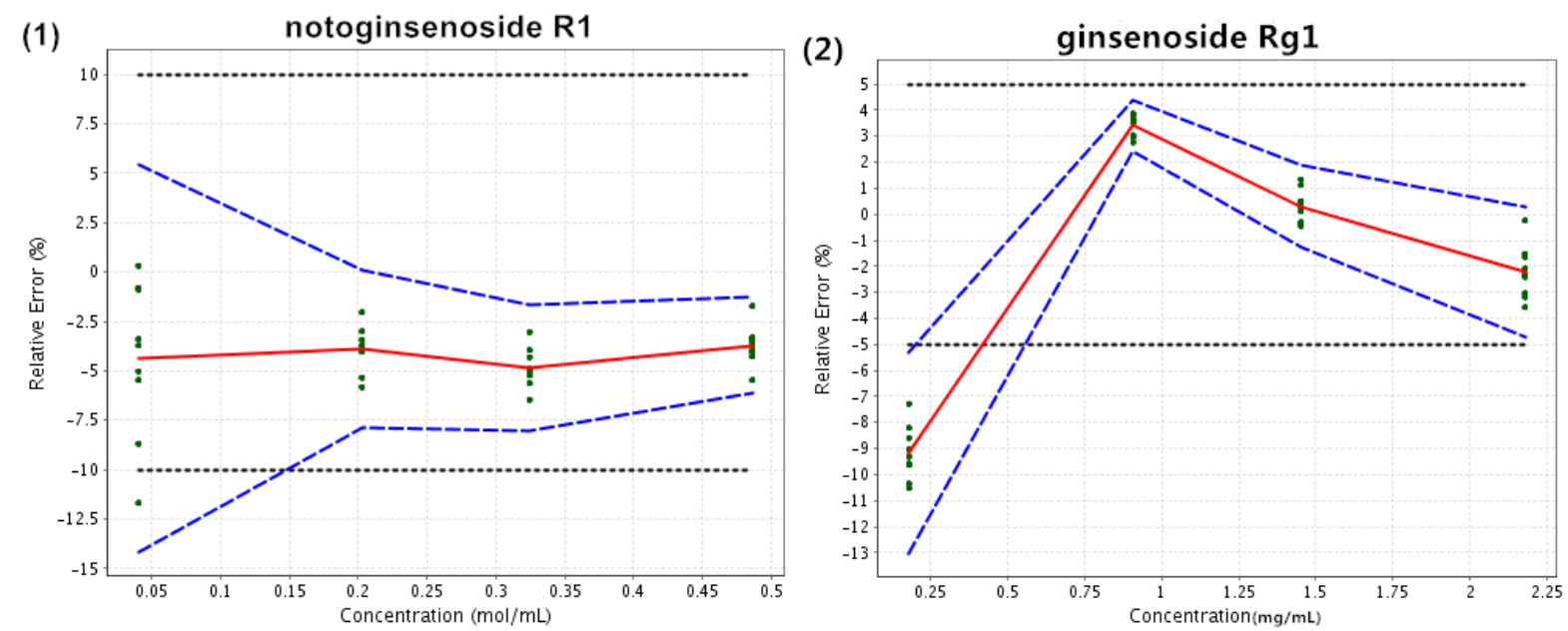

(3)

ginsenoside $\mathrm{Re}$

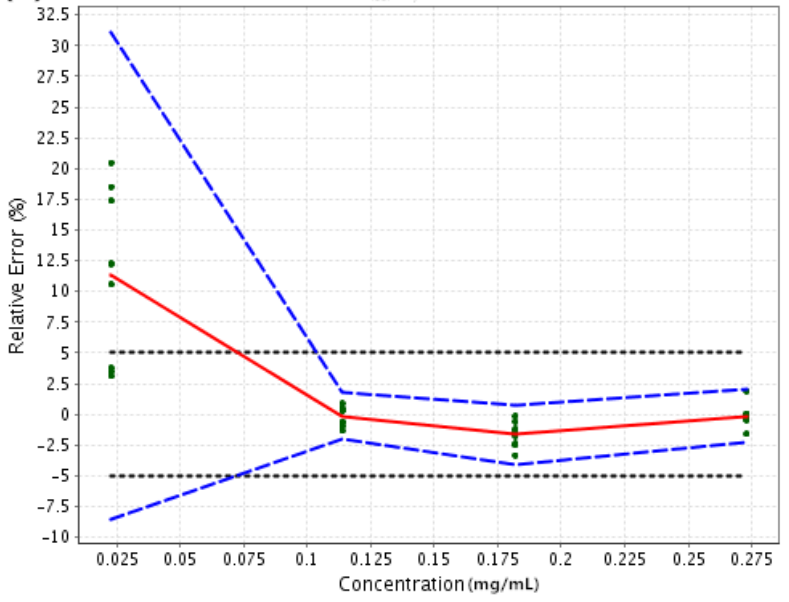

(4)

ginsenoside $\mathbf{R b} 1$
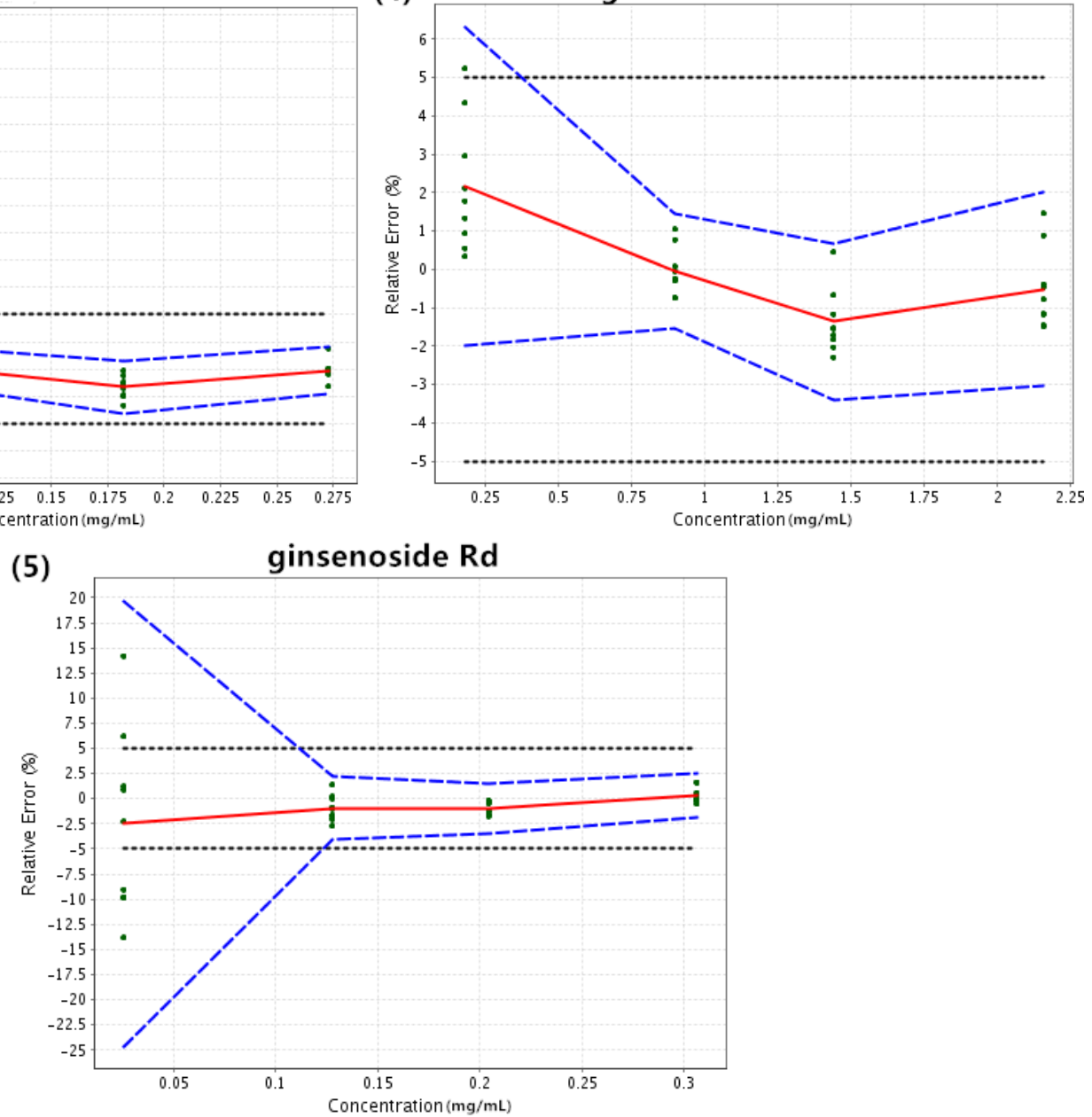

Fig. (3). Accuracy profiles of the 5 components. The plain line is the relative bias. The dashed lines are the $90 \% \beta$-expectation tolerance limit and dotted lines represent the acceptance limit. The acceptance limit of the first component is $\pm 10 \%$ and the rest of them are $\pm 5 \%$.

\section{REFERENCES}

B. Debrusa, P. Lebruna , A. Ceccatob, "Application of new methodologies based on design of experiments, independent component analysis and design space for robust optimization in liquid chromatography," Analytica Chimica Acta, vol. 691, pp. 33-42, Feb. 2011
[2] D. Awotwe-Otoo, C. Agarabia, P. J. Faustinoa, "Application of quality by design elements for the development and optimization of an analytical method for protamine sulfate," Journal of Pharmaceutical and Biomedical Analysis, vol. 62, pp. 61-67, Jan. 2012.

[3] M. H. Rafamantanana, B. Debrus, G. E. Raoelison, “Application of design of experiments and design space methodology for the HPLC-UV separation optimization of aporphine alkaloids from leaves of Spirospermum penduliflorum Thouars," Journal of 
Pharmaceutical and Biomedical Analysis, vol. 62, pp. 23-32, Jan. 2012.

[4] International Conference on Harmonization of technical requirements for registration of pharmaceuticals for human use (ICH), ICH Q8 (R2): Pharmaceutical Development, pp. 1-24, Aug. 2009. Available from: http://www.ich.org/f i leadmin/Publ ic_Web_Site/ICH_Products/Guidelines/Quality/Q8_R1/Step4/Q8_ R2_Guideline.pdf.

[5] M. Feinberg, "Validation of analytical methods based on accuracy profiles," Journal of Chromatography A, vol. 1158, pp. 174-183, Feb. 2007.

[6] P. N. Brown, P. Lister, "Current initiatives for the validation of analytical methods for botanicals," Current Opinion in Biotechnology, vol. 25, pp. 124-128, Dec. 2013.
[7] E. Rozet, V. Wascotte, N. Lecouturier, "Improvement of the decision efficiency of the accuracy profile by means of a desirability function for analytical methods validation," Analytica Chimica Acta, vol. 591, pp. 239-247, Apr. 2007.

[8] Guidance for Industry, Bioanalytical Method Validation, US Department of Health and Human Services, Food and Drug Administration, Center for Drug Evaluation and Research (CDER), Center for Biologics Evaluation and Research (CBER), Rockville, May 2001.

[9] P. Hubert, J. J. Nguyen-Huu, B. Boulanger, "Harmonization of strategies for the validation of quantitative analytical procedures A SFSTP proposal - Part II," Journal of Pharmaceutical and Biomedical Analysis, vol. 45, pp. 82-96, Jul. 2007.

(C) Dai et al.; Licensee Bentham Open.

This is an open access article licensed under the terms of the Creative Commons Attribution Non-Commercial License (http://creativecommons.org/licenses/ by-nc/3.0/) which permits unrestricted, non-commercial use, distribution and reproduction in any medium, provided the work is properly cited. 